This is a postprint version of the following published document:

Crespo, M., Méndez, N., González, M., Baselga, J. y Pozuelo, J. (2014): Synergistic effect of magnetite nanoparticles and carbon nanofibres in electromagnetic absorbing composites. Carbon, 4, pp. 63-72.

DOI: $\underline{10.1016 / \text { j.carbon.2014.02.082 }}$

(C) Elsevier, 2014

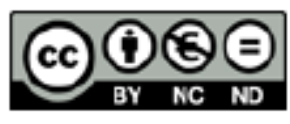

This work is licensed under a Creative Commons Attribution-NonCommercialNoDerivatives 4.0 International License. 


\title{
Synergistic effect of magnetite nanoparticles and carbon nanofibres in electromagnetic absorbing composites
}

\author{
María Crespo, Néstor Méndez, María González, Juan Baselga, Javier Pozuelo* \\ Departamento de Ciencia e Ingeniería de Materiales e Ingeniería Química (IAAB), \\ Universidad Carlos III de Madrid, 28911 Legane's, Madrid, Spain
}

\begin{abstract}
Epoxy composites with different amounts of magnetite nanoparticles, carbon nanofibres (CNF) and magnetite decorated CNF were prepared and characterized. A simple method for the magnetite CNF decoration was developed by adsorbing preformed oleic acid capped magnetite nanoparticles over the CNF surface. A synergy between magnetite nano particles and CNF was found to have crucial effects in the electromagnetic shielding effi ciency of the prepared materials. This effect has been analysed by their electrical conductivity in terms of percolation theory and complex permittivity at high frequencies. Electromagnetic shielding mechanisms (reflection, absorption and transmission) were individually studied in the $118 \mathrm{GHz}$ range. Results show that decoration of CNF with mag netite, notably increases permittivity and high frequency AC conductivity and enhances the electromagnetic shielding efficiency up to around $20 \mathrm{~dB}$ at high frequencies. It is pro posed that interfacial polarization adds an additional dissipation mechanism that may be responsible for the observed electromagnetic shielding enhancement.
\end{abstract}

\section{Introduction}

An electronic device is considered compatible with the envi ronment when its emissions do not affect other devices and is not affected itself by external emissions. Thus, any effort for minimizing interferences and protecting electronic de vices is of prime importance. Electromagnetic interferences (EMI) in many cases are minimized through the circuit design or using filters, while protection of devices is generally im proved with the use of metallic coatings [1 3], metal casings or polymer conductive composites [4 23]. The shielding mechanism in the two former cases is mainly related with radiation reflection processes while radiation absorption is the main mechanism in the latter. The choice of a material for shielding depends strongly on the final application: for example, low reflection losses and high absorption losses are required for military radar shielding materials whilst lightweight materials are a must for aerospace shielding applications; polymer matrix composites are thus promising materials that may fulfil a variety of requirements in all the above emerging fields.

Three mechanisms for electromagnetic shielding are com monly accepted: reflection, absorption and multiple reflec tions [22]. In good conductors (i.e.: metals), the most important contribution is reflection, where losses are related with the ratio between conductivity and permeability $(\sigma / \mu)$. When considering composites, absorption, which depends on the product $(\sigma \cdot \mu)$ and on the thickness of the material, is

* Corresponding author: Fax: +34 91 6249430. E mail address: jpozue@ing.uc3m.es (J. Pozuelo). 
the main mechanism. The third mechanism (multiple reflec tions) is related to the conductor skin thickness and for high frequencies, in the $\mathrm{GHz}$ range, is negligible [23]. For the pro tection of electronic devices towards external radiation, the most important mechanism should be reflection, so high con ductivity and low permeability is required. On the other hand, if the application is focused on dissipating radiation as heat, low reflection and high absorption are needed instead. Although the concept of electromagnetic shielding is quite clear, there is certain controversy regarding the parameters used for describing this phenomenon. Some authors only consider the total electromagnetic losses and do not consider reflection and absorption losses separately. This assumption may lead to inaccurate conclusions since artefacts related to the sample thickness may be interpreted as strong absorption processes taking place at well defined frequencies. Thus, a strict differentiation of electromagnetic shielding mecha nisms is essential for the development of a new material.

Carbon nanofibres (CNF) and carbon nanotubes (CNT) have been incorporated in different polymer matrices since the early eighties, mainly because of their high electric conduc tivity and aspect ratio which enables the preparation of com posites with good conductivity and electromagnetic shielding properties using low filler quantities. CNF have been intro duced in a wide variety of polymers, from polypropylene foams [5] to liquid polymer crystals [6] among others. They have been also combined with magnetic particles by electros pinning [7,8], reaching $3040 \mathrm{~dB}$ shielding efficiency in the fre quency range of $14 \mathrm{GHz}$ and showing the influence of magnetic particles in electromagnetic losses. An excellent re view on CNF/polymer composites focused on electrical prop erties, electromagnetic shielding and thermal properties has been reported by Al Saleh et al. [4]. CNT have been also widely used in many polymer matrices such as: polyurethanes, where $20 \%$ CNT gave losses close to $20 \mathrm{~dB}$ [9]; polystyrene, with losses lower than $12 \mathrm{~dB}$ in the $\mathrm{X}$ band and being reflec tion the most important contribution $[10,11]$; polycarbonate, with losses close to $20 \mathrm{~dB}$ with $5 \%$ of $\mathrm{CNT}$, being absorption the most important contribution [12]; PVA/PAAc, with very high absorption with a CNT content up to $72 \%$ [13]. Even CNT, CNF and magnetic particles combinations, have been prepared for improving electromagnetic shielding properties [19 21].

Although CNF or CNT composites, preparation and charac terization with thermosetting polymers and phenolic or DGE BA based epoxies, has been previously reported in the literature [14 18] in this work we present interesting results that arise when combining CNF decorated with magnetite nanoparticles in an epoxy matrix. Among the different epoxy formulations, we have selected a hydrogenated derivative of diglycidyl ether of bisphenol A (HDGEBA) epoxy resin, whose main feature is the absence of phenyl groups in its structure while keeping good reactivity towards common amine based curing agents. Its low polarity and the presence of flexible cyclohexyl groups instead of rigid aromatic rings makes the viscosity of HDGEBA appreciably lower than standard DGEBA epoxy based resins; this fact joined with moderate glass transition temperatures makes this selection adequate for coatings, shaping complex components and encapsulation
[24 28] of electronic devices in applications where tempera ture requirements are not severely stringent.

In this work thermosetting materials containing $110 \%$ (w/w) of CNF or magnetite nanoparticles are prepared and their behaviour is compared with that of composites contain ing magnetite decorated CNF in a 50:50 weight ratio. As it will be shown, decoration of CNF with magnetite introduces some kind of synergism that is absent when both particles are topo logically separated. The electromagnetic shielding mecha nisms operating in each composite material are studied with the aim of preparing panels with suitable electromag netic absorption properties in the range $118 \mathrm{GHz}$, where most of the developing telecommunication applications need an improvement.

\section{Experimental}

\subsection{Materials and characterization measurements}

Carbon nanofibres (CNF) were kindly supplied by Grupo Anto lín S.A. (Spain). Nominal properties of CNF as provided by the manufacturer where: $\sigma \approx 10^{3} \mathrm{~S} / \mathrm{m}$, diameter $2080 \mathrm{~nm}$, length $>30 \mu \mathrm{m}$. Magnetite nanoparticles were in house synthesized starting from $\mathrm{FeCl}_{2} \cdot 4 \mathrm{H}_{2} \mathrm{O}, \mathrm{FeCl}_{3} \cdot 6 \mathrm{H}_{2} \mathrm{O}, \mathrm{NH}_{4} \mathrm{OH}(28 \% \mathrm{v} / \mathrm{v})$ and oleic acid which were purchased from Sigma Aldrich and used without any further purification. The hydrogenated derivative of diglycidyl ether of bisphenol A (HDGEBA) epoxy resin was supplied by CVC Specialty Chemicals (USA); its epoxy equivalent mass was $210 \mathrm{~g} \mathrm{~mol}^{1}$ determined by acid titration. m Xylilenediamine (Sigma Aldrich) was used as curing agent. Tetrahydrofuran (THF) was purchased from Sig ma Aldrich.

\subsection{Surface modification of CNF with magnetite nanoparticles}

Magnetite nanoparticles (Mag), covered with oleic acid, were prepared and characterized in our laboratories in a previous work [29]. In a typical experiment, $14.5 \mathrm{~g}$ of $\mathrm{FeCl}_{3} \cdot 6 \mathrm{H}_{2} \mathrm{O}$ and $5.37 \mathrm{~g}$ of $\mathrm{FeCl}_{2} \cdot 4 \mathrm{H}_{2} \mathrm{O}$ were dissolved in $300 \mathrm{~mL}$ of deionised water in a round bottom flask placed in an ultrasonic bath with mechanical stirring at $7080^{\circ} \mathrm{C}$. $25 \mathrm{~mL}$ of $25 \% \mathrm{NH}_{4} \mathrm{OH}$ were quickly added to the solution. After a few seconds the solution turned black (typical magnetite colour) and $10 \mathrm{~mL}$ of oleic acid were added to the suspension stirring vigorously for $2 \mathrm{~h}$. The black fine magnetite precipitate was separated from the solution using a magnet and washed several times with hot deionised water and acetone to remove non reacted metallic salts and excess of oleic acid respectively. The clean precipitate was vacuum dried at room temperature for $24 \mathrm{~h}$.

Carbon nanofibres decorated with magnetite nanoparti cles (CNF:Mag) were prepared by direct incorporation of pre formed oleic acid covered magnetite nanoparticles onto the CNF surface. In a typical experiment, appropriate amounts of CNF and magnetite nanoparticles were separately dis persed in heptane by sonication and mechanical stirring. Both dispersions were then mixed in a 1:1 mass ratio and son icated again for $10 \mathrm{~min}$ to ensure adsorption of magnetite over the CNF. Modified nanofibres were removed from the 
dispersion using a magnet leaving a completely clear and transparent supernatant, indicating that all magnetite was adsorbed onto the CNF. The precipitate was finally dried in vacuum at room temperature for $1 \mathrm{~h}$.

\subsection{Composite preparation}

Three different composites were prepared: epoxy/Mag, epoxy/ CNF, and epoxy/CNF:Mag. The amount of CNF or magnetite in the two former composites was: $1 \%, 2.5 \%, 5 \%$ and $10 \% \mathrm{w} / \mathrm{w}$. The amount of CNF:Mag was selected to give equivalent total amount of Mag or CNF as in the epoxy/magnetite and epoxy/ CNF composites: $2.5 \%, 5 \%, 10 \%$ and $20 \%$; for example, a $10 \%$ CNF:Mag composite contained $5 \%$ of either magnetite and CNF. Assuming volume additivity, mass fractions were trans formed into volume fractions using the expression: $\phi_{i}\left[1+\frac{\left(1 \omega_{i}\right) \rho_{i}}{\omega_{i} \rho_{e}}{ }^{1}\right.$ where $\omega_{i}$ and $\rho_{i}$ refer to the mass fractions and density of either magnetite, CNF or CNF:Mag and $\rho_{e}$ is the density of epoxy $\left(\rho_{e} \quad 1.25 \mathrm{~g} \mathrm{~cm}^{3}\right)$; magnetite nanoparti cles density, was calculated from the known densities of crys talline magnetite $\left(5.15 \mathrm{~g} \mathrm{~cm}^{3}\right)$ and oleic acid $\left(0.895 \mathrm{~g} \mathrm{~cm}^{3}\right)$, and the mass fraction of oleic acid as measured by TGA [29], giving a value of $2.6 \mathrm{~g} \mathrm{~cm}^{3}$; density of CNF was $1.97 \mathrm{~g} \mathrm{~cm}^{3}$ as communicated by the supplier; density of CNF:Mag was calculated with the following expression. $\rho_{\text {CNT:Mag }}$ $2 /\left[\rho_{\mathrm{CNF}}^{1}+\rho_{\mathrm{Mag}}^{1}\right]$.

For the preparation of nanocomposites, both the filler and HDGEBA were blended with THF and appropriate amounts of the mixtures were placed in a glass vial, mechanically stirred and sonicated at room temperature giving stable suspen sions; solvent removal (vacuum, $80^{\circ} \mathrm{C}$ ) prior curing did not af fect stability of the suspensions and stable dispersions in HDGEBA were obtained. Dispersions were mixed with stoichi ometric amounts of $\mathrm{m}$ xylilenediamine curing agent and cured at $90^{\circ} \mathrm{C}$ for $1 \mathrm{~h}$ and post cured $2 \mathrm{~h}$ at $130^{\circ} \mathrm{C}$ to ensure full conversion.

\subsection{Techniques}

The pristine CNF, the magnetite nanoparticles and the modi fied nanofibres were characterized by Transmission Electron Microscopy (TEM, 200 kV Philips Tecnai 20) and Wide Angle $\mathrm{X}$ ray Diffraction (XDR, Panalytical X'pert Pro X ray diffrac tometer with $\mathrm{Cu} \mathrm{K} \alpha$ radiation $(\lambda \quad 0.15406 \mathrm{~nm})$ ). SEM images of the cured cryo fractured specimens were obtained with a Philips XL 30 scanning electron microscope. Magnetic proper ties of CNF:Mag particles were investigated by Vibrating Sam ple Magnetometry (VSM, CFMS Cryogenic Ltd.) at $300 \mathrm{~K}$ with a $10 \mathrm{~T}$ magnetic field. Electrical properties of the composites were evaluated using a HP 34401A device with $100 \mu \Omega$ resolu tion and $10 \mathrm{G} \Omega$ of upper limit. Measurements were performed in 4 wire DC configuration to obviate the electrical resistance of the wires. The temperature of the samples during the mea surement was $28.8^{\circ} \mathrm{C}$. Samples were prepared placing a drop of the uncured blend was between two steel plates (diame ters: 2.4 and $3 \mathrm{~cm}$ ) and cured using the usual schedule. The thickness of the sample was controlled using $100 \mu \mathrm{m}$ Teflon spacers placed between the steel disks. Shielding was evalu ated using a two port Agilent ENA Network Analyzer (E5071) in the range of $118 \mathrm{GHz}$ with an Agilent $7 \mathrm{~mm}$ coaxial trans mission line adapted to the Network Analyzer.

\subsubsection{EMI shielding}

The global EMI shielding efficiency, $\mathrm{SE}_{\mathrm{T}}$, can be quantified as the sum of the contributions of the three shielding mecha nisms: reflection, absorption and multiple reflections, as shown in Eq. (1).

$$
\mathrm{SE}_{\mathrm{T}} 20 \log \left(\frac{\eta_{0}}{4 \eta_{\mathrm{S}}}\right)+20 \log \left(\exp \left(\frac{2 d}{\delta}\right)\right)+20 \log \left(1 \exp \left(\frac{2 d}{\delta}\right)\right)
$$

where $\eta_{0}$ and $\eta_{S}$ are the intrinsic impedances of air and the sample; $d$ is the sample thickness and $\delta$ is the skin thickness of the material, which is dependent on frequency, $f$, magnetic permittivity, $\mu$, and on conductivity, $\sigma$, according to $\delta \quad(\pi f \mu \sigma)^{1 / 2}$.

The first term of Eq. (1) corresponds to the reflection losses which only depend on the impedances, while the second and third terms depend on the thickness of the material and the skin. The third term can be neglected when the thickness of the material is considerably higher than the conductor skin. Under these conditions, according to Al Saleh et al. [23], Eq. (1) can be re written as a function of conductivity, $\sigma$, and per meability, $\mu$, as:

$\mathrm{SE}_{\mathrm{T}} \quad \mathrm{SE}_{\mathrm{R}}+\mathrm{SE}_{\mathrm{A}} \quad\left(39.5+\frac{10 \log \sigma}{2 \pi f \mu}\right)+(8.7 d \sqrt{\pi f \mu \sigma})$

It is worthy to note that the reflection term depends on $(\sigma / \mu)$ and not on the thickness, while the absorption term is a function of $(\sigma \cdot \mu)$ and the sample thickness. These contribu tions to shielding may be determined through the measure ment of the scattering parameters, $S_{11}$ and $S_{21}$, with a vector network analyzer (ENA). An ENA instrument measures the transmitted and reflected power $\left(P_{T}\right.$ and $\left.P_{R}\right)$ when a material is irradiated with an incident power $P_{I}$. The ratios between the scattering parameters and the transmission, reflection and absorption SE are given by the following equations.

$$
\begin{aligned}
& \mathrm{SE}_{\mathrm{T}} \quad 10 \log \frac{\mathrm{P}_{\mathrm{I}}}{\mathrm{P}_{\mathrm{T}}} \quad 10 \log \frac{1}{\left|\mathrm{~S}_{21}\right|^{2}} \\
& \mathrm{SE}_{\mathrm{R}} \quad 10 \log \frac{\mathrm{P}_{\mathrm{I}}}{\mathrm{P}_{\mathrm{I}} \quad \mathrm{P}_{\mathrm{R}}} \quad 10 \log \frac{1}{1\left|\mathrm{~S}_{11}\right|^{2}} \\
& \mathrm{SE}_{\mathrm{A}} \quad 10 \log \frac{\mathrm{P}_{\mathrm{I}} \quad \mathrm{P}_{\mathrm{R}}}{\mathrm{P}_{\mathrm{T}}} \quad 10 \log \frac{1\left|\mathrm{~S}_{11}\right|^{2}}{\left|\mathrm{~S}_{21}\right|^{2}}
\end{aligned}
$$

The scattering parameters (S parameters) were measured and used to calculate the complex magnetic permeability and dielectric permittivity of all the prepared samples. The measurements were performed according to the transmis sion/reflection method using a two port Agilent ENA Network analyzer (E5071), adapted with a $7 \mathrm{~mm}$ coaxial transmission line, in the frequency range from 1 to $18 \mathrm{GHz}$. Samples cured in silicon molds were machined to the final required geome try for the coaxial line: toroids of nominal internal and exter nal diameters close to 3.04 and $7 \mathrm{~mm}$, respectively and thickness in the range of $113 \mathrm{~mm}$. Using the built in soft ware, a geometry correction was applied for small deviations from nominal geometry. 


\section{Results and discussion}

\subsection{Structural characterization}

The size of the oleic acid capped magnetite nanoparticles was characterized by Transmission Electron Microscopy (TEM), $\mathrm{X}$ ray Diffraction (XRD) and magnetic measurements in a pre vious work [29] yielding values of: 9.4, 9.4 and $9.3 \mathrm{~nm}$, respec tively. Superparamagnetic behaviour was confirmed by hysteresis loops at 2 and $300 \mathrm{~K}$ and a blocking temperature of $110 \mathrm{~K}$ was observed. This superparamagnetic behaviour is retained when magnetite nanoparticles are grafted to the sur face of CNF (see supplementary information). No significant changes could be observed by the presence of CNF.

TEM images of Pristine CNF and CNF:Mag are shown in Fig. 1. Pristine fibres show a wide size distribution and a large variety of shapes ranging from almost straight to highly con torted. Images of the decorated nanofibres confirm that the $9.4 \mathrm{~nm}$ diameter magnetite nanoparticles are adsorbed on the surface of CNF, although the coverage is not uniform along the fibres.

Powder XRD was recorded for the synthesized materials to confirm the preservation of the original crystalline structure from both the as prepared magnetite nanoparticles and the pristine CNF. The XRD pattern (Fig. 2) of CNF shows two in tense peaks at scattering angles of 26.1 and 44.25 which can be indexed to the (002) and (101) planes of a hexagonal graphite lattice respectively. These peaks remain unmodified in CNF:Mag. The magnetite nanoparticles show a typical spi nel like structure evidenced by the signals at 30.2, 35.6, 43.5, $53.8,57.4$ and 63.0 that can be attributed to the (220), (311), (400), (422), (511) and (440) crystalline planes respectively. Neither other phases (such as hematite) nor changes in the diffraction pattern were observed, indicating that the experi mental procedure for the incorporation of the nanoparticles onto the fibres do not have any appreciable effect on their crystalline structure.

SEM images on cryogenically fractured fully cured speci mens reveal a uniform dispersion of decorated CNFs (see Sup porting information).

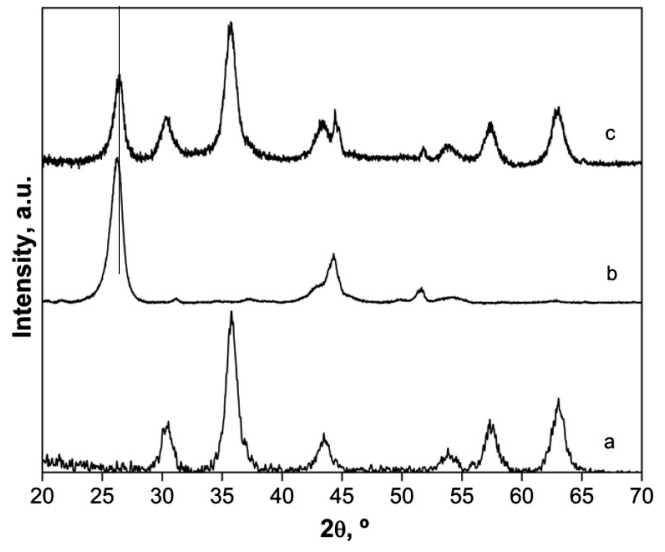

Fig. 2 - X-ray diffractograms of magnetite nanoparticles (a), pristine CNF (b), CNF:Mag (c).

\subsection{Electromagnetic characterization}

\subsubsection{DC electrical conductivity}

Results on DC electrical conductivity of the composites with CNF and CNF:Mag are shown in Fig. 3. The conductivity of the neat epoxy is below the detection limit of the instrument used in these measurements, since it is an insulating material $\left(\sigma_{\mathrm{e}} \approx 10{ }^{15} \mathrm{~S} / \mathrm{m}\right)$. The values obtained for the composites with decorated and non decorated nanofibres are quite high and increase with CNF concentration ranging between $10^{6}$ (typi cal range for insulating materials) to $0.2 \mathrm{~S} / \mathrm{m}$ (typical of semi conductors) for $1 \% \mathrm{w} / \mathrm{w}$ and $10 \%$, respectively. A similar trend in conductivity was observed for CNF:Mag composites, but values were one order of magnitude below the one observed for pristine CNF. The high conductivity values of composites containing the lowest CNF and CNF:Mag concentrations, indi cate that even at low loading, the system is beyond the perco lation threshold. The percolation threshold is related to the contact between fibres and depends strongly on their aspect ratio and dispersion degree, making difficult to compare re sults from different authors: reported values for CNF polymer composites may be between $7 \%$ and $8 \%$ [30] or $0.5 \%$ and $1 \%$

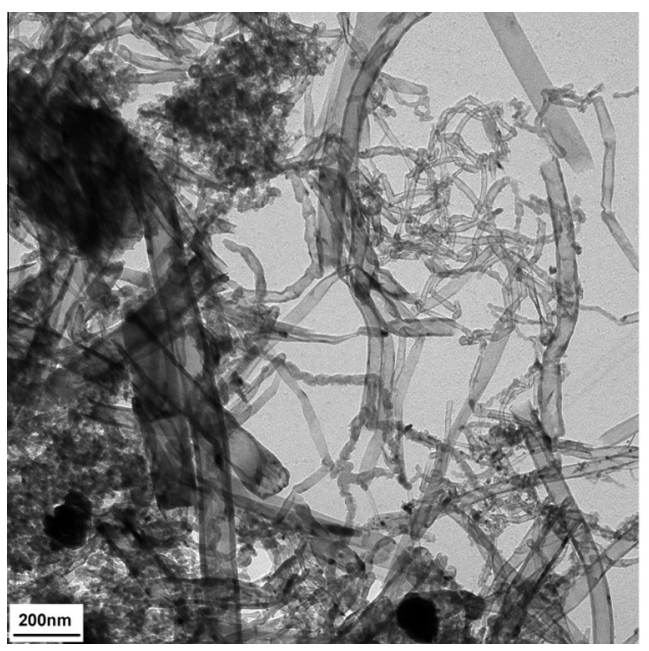

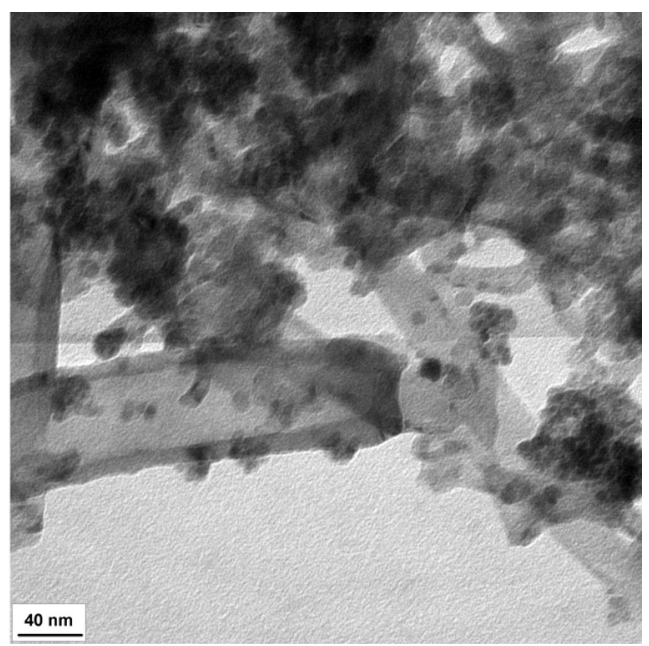

Fig. 1 - TEM of pristine CNF (left) and CNF:Mag (right). 


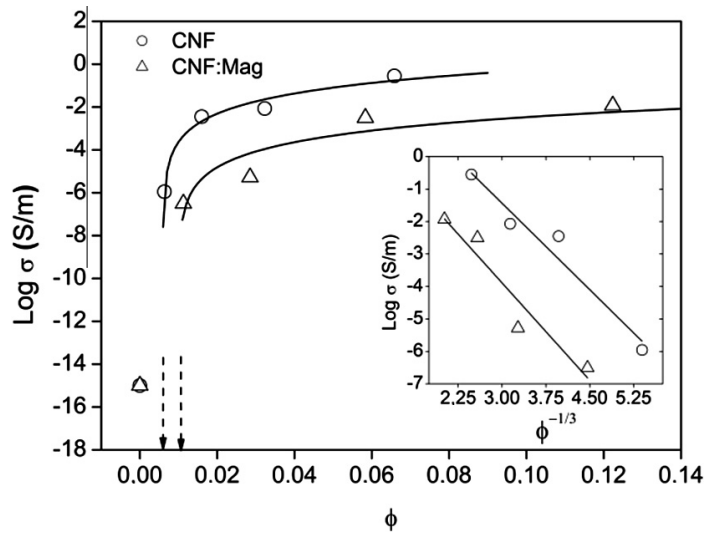

Fig. 3 - DC conductivity of CNF (s) and CNF:Mag (D) composites as a function of filler volume fraction. Solid lines are the best fit to $r /$ $(/-/ \mathrm{C})^{\mathrm{t}}$. Inset: DC conductivity as a function of $/^{-1 / 3}$ (see text for details).

(w/w) [31]. Nevertheless, this discrepancy may be avoided by analysing the data in terms of the percolation theory [32] hav ing the conductivity as a function of the volume fraction $(\phi), \sigma$ $\propto\left(\begin{array}{ll}\phi & \phi_{C}\end{array}\right)^{t}$, from which a percolation threshold of $0.6 \%$ vol. for CNF and 0.1\% vol for CNF:Mag (Fig. 3) was obtained. The expo nent $t \sim 2.2$ was the same for both fillers within the experi mental error, being in accord with reported values for other polymer/conductor composites [33].

The fact that both fillers, pristine and magnetite decorated CNF, present a very similar percolation threshold indicates that adsorbed magnetite does not appreciably alter the dis persion process of the filler.

Conductivity in similar polymer/conductor disordered systems has been explained in terms of fluctuation induced tunnelling conduction theory [33 35]. In this theoretical framework, electrical conduction is dominated by electron transfer across the insulating gaps between conducting $\mathrm{fi}$ bres or clusters. Conductivity is therefore controlled by the junction gap width, $\omega$, and the theory predicts $\log \sigma \propto$ $\omega$ if the conducting filler is uniformly dispersed in the insulating matrix. Although it is not the purpose of this pa per to study in detail the transport mechanism, a sugges tive result arises when $\log \left(\sigma_{D C}\right)$ is plotted against $\phi^{1 / 3}$ as depicted in Fig. 3 (inset); following the work of Connor et al. [[33]and Refs. contained therein], the gap width of fil ler filler junctions must be proportional to $\phi^{1 / 3}$ and, as a consequence $\log \sigma$ should vary linearly with $\phi^{1 / 3}$. The lin ear plots observed in Fig. 3, that show the same slope with in experimental uncertainty, suggest therefore that fluctuation tunnelling may be the underlying electron con duction mechanism for the composites prepared in this work, thus being the gap width similar in both decorated and not decorated composites. Additionally, the lower inter cept found for the CNF:Mag composites, suggests a reduced junction contact area. It seems therefore, that the oleic acid capped magnetite particles adsorbed on the CNF do not modify the contact width, but reduces the surface portion of the fibres or clusters available for electron tunnelling. This fact is in well agreement with the TEM observations (Fig. 1) discussed above.
3.2.2. Dielectric permittivity and magnetic permeability of epoxy/Mag composites

Interaction of a material with electromagnetic fields can be analysed in terms of the complex permittivity $(\varepsilon)$ and perme ability $(\mu)$, reflecting the interaction with the electric and the magnetic parts of the electromagnetic field, respectively. These magnitudes are described by an imaginary component $\left(\varepsilon^{\prime \prime}\right.$ or $\left.\mu^{\prime \prime}\right)$ which are related to the energy loss and a real com ponent $\left(\varepsilon^{\prime}\right.$ or $\left.\mu^{\prime}\right)$ which gives information about the stored en ergy. The ratio between both components is the loss factor $\left(\varepsilon^{\prime \prime}\right)$ $\varepsilon^{\prime}$ or $\left.\mu^{\prime \prime} / \mu^{\prime}\right)$. The values of the real magnetic permeability of the nanocomposites (see supplementary information) are very close to one and the values of the imaginary part are close to zero in all cases, indicating that the effect of the reinforce ment is mainly attributed to its interaction with the electric field.

The real component of the dielectric permittivity, $\varepsilon^{\prime}$, and the dielectric loss tangent, $\tan \delta$, for epoxy composites con taining different amounts of magnetite are presented in Fig. 4 as a function of frequency. Superimposed over a general decreasing trend, some maxima and minima appear. These are experimental artefacts are associated to the sample thick ness and neither to multiple reflections nor to epoxy or mag netite relaxation processes in this frequency range [36 39].

The effect of magnetite nanoparticles on the dielectric properties of the composites is small: only a slight increase in $\varepsilon^{\prime}$ with magnetite concentration is observed (at $1 \mathrm{GHz}$, from $\approx 3$ for the neat epoxy to $\approx 3.4$ for the composite with $10 \% \mathrm{w} / \mathrm{w}$ of magnetite, which corresponds to a volume fraction of 0.051 ) while loss tangent remains constant and very close to zero (0 0.04), for all compositions and frequencies, indicating that losses are negligible. This result reflects a weak interac tion with the electric field and between the electric dipoles of the nanoparticles that can be attributed to the lack of con nectivity between the particles even at 0.05 volume fraction.

This is in reasonable agreement with the work of Ali zade [40] that states that below volume fractions around 0.03 , for magnetite particles with similar size as the ones used in this work, interactions between electric dipoles of nanoparticles can be neglected. It is worthy to note that the percolation limit reported for hard core spherical particles of $\approx 10 \mathrm{~nm}$
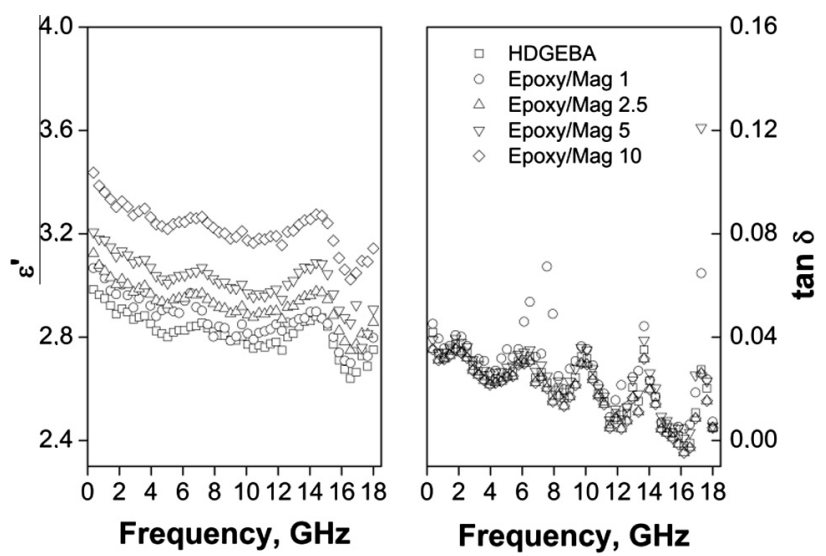

Fig. 4 - Real permittivity and losses tangent for epoxy matrix (h) and Epoxy/Mag composites with composition 1\%(s), 2.5\% (4), 5\% (5) and $10 \%(\mathrm{e})$. 
diameter is around 16\% v/v [41 43], that is far above the max imum amount used in this work $(5.0 \% \mathrm{v} / \mathrm{v})$. Therefore, the effective permittivity of the epoxy/magnetite composites should follow a simple rule of mixtures. Within the several equations that have been proposed for non interacting particles below the percolation limit [44] we have selected the Cummings equation because of its simplicity: $\log \left(\varepsilon_{\text {eff }}\right) \quad \sum \phi_{i} \log \left(\varepsilon_{i}\right)$, where $\phi_{i}$ and $\varepsilon_{i}$ are the volume fraction and permittivity of the $i$ th component. Using this equation, we have extracted the permittivity of magnetite which results to be $37.8 \pm 3.1$ in the frequency range $3.6 \times 10^{8} 1.7 \times 10^{10} \mathrm{~Hz}$, in excellent agreement with reported values in a narrower frequency range [45].

3.2.3. Dielectric permittivity, loss tangent and high frequency AC conductivity of epoxy/CNF and epoxy/CNF:Mag) composites When composites containing either CNF or CNF:Mag are ana lysed, a strong dependence of both $\varepsilon^{\prime}$ and $\sigma$ (calculated as $\left.\sigma \quad 2 \pi f \varepsilon_{0} \varepsilon^{\prime \prime}\right)$ with the amount of nanoparticles and frequency is observed and much higher values than those for the Epoxy/Mag system are obtained. Results are presented in Fig. 5 in the form of double logarithmic plots.

Conductivity of CNF is typically below $10^{3} \mathrm{~S} / \mathrm{m}$ and permit tivity is usually taken as $1215[46,47]$ due to their structural similarity with graphite. As expected, calculated values of permittivity, assuming a simple rule of mixtures for indepen
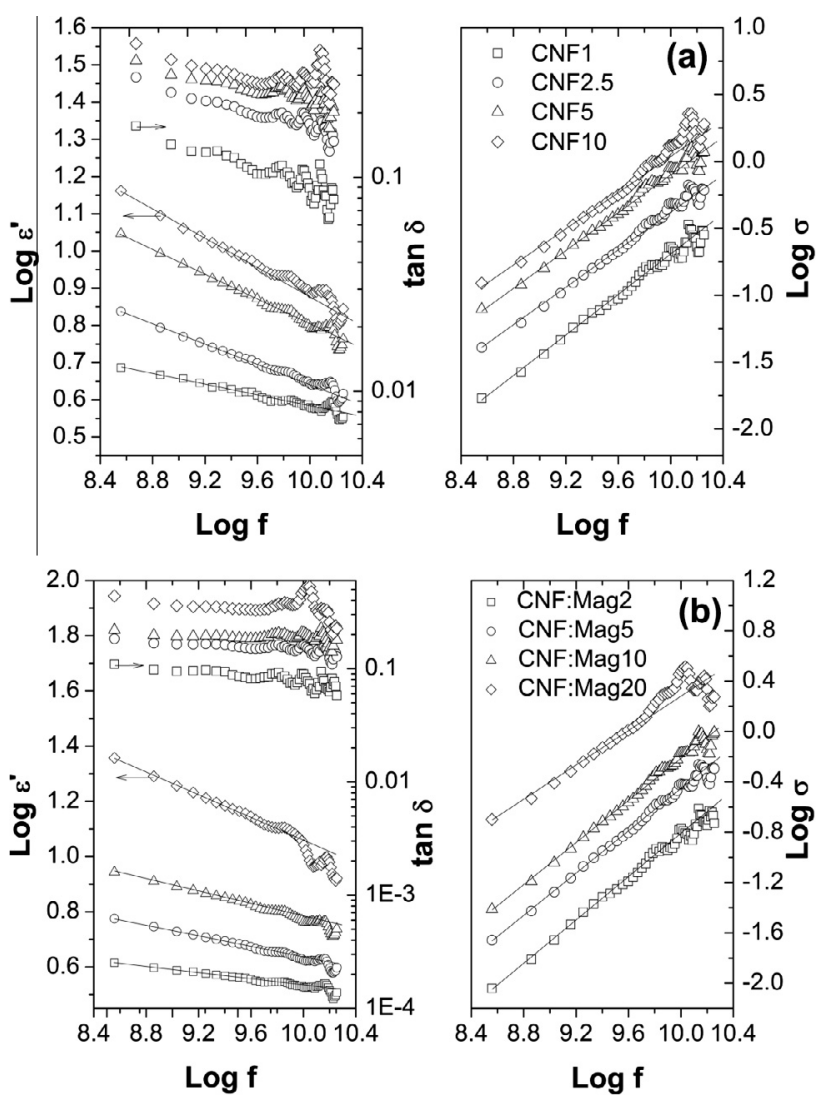

Fig. 5 - Double logarithmic plots of real permittivity $\mathrm{e}^{0}$ (left), and conductivity r (right) for: (a) Epoxy/CNF, and (b) Epoxy/CNF:Mag composites. Mass compositions are indicated in the inset. Lines correspond to the best linear fits of the initial portion of the curves. dent non interacting particles (not shown), are systematically lower than the experimental data and the difference between both increases with frequency; this is a clear indication of strong inter particle interactions due to their connectivity since all the studied systems are above the percolation threshold.

The frequency dependence of conductivity is typically ex plained in terms of the percolation theory in fractal structures $[33,48]$, which considers $\sigma \propto f^{x}$ and $\varepsilon \propto f^{y}$, where the expo nents follow the general relation $x+y \quad 1[49,50]$. Linear fits of the first initial portions of the double logarithmic plots in Fig. 5( $a$ and $b$ ) yield the following averaged values for both CNF and CNF:Mag systems: $x \quad 0.81 \pm 0.09$ and $y \quad 0.12 \pm 0.06$ in well agreement with the theory. When comparing CNF and CNF:Mag composites it becomes clear that at low load ings, CNF present higher conductivity and permittivity than CNF:Mag, in accordance with the DC conductivity measure ments presented previously. But the conductivity for the CNF:Mag composite with the highest loading $(20 \% \mathrm{w} / \mathrm{w})$ at the lowest measured frequency $\left(3.6 \times 10^{8} \mathrm{~Hz}\right)$, DC and AC con ductivities of CNF:Mag composite are very similar, within experimental error $(0.120 .2 \mathrm{~S} / \mathrm{m})$, indicating that for this sys tem, the critical frequency, i.e., the frequency below which conductivity is frequency independent, must be close to the lowest limit measured with the network analyser.

Dielectrics are known to have two foremost energy loss mechanisms, the first being the conduction loss, occurring at low frequencies, where conduction is mainly dominated by the materials resistance, and the dielectric loss, generated by the induced polarization when the AC field interacts with the material. In the latter case, the loss can either be by elec tron polarization, ion polarization or electric dipolar polariza tion. Electron and ion polarization are relatively weak at the microwave region and tend to appear beyond the infrared range [51]. Consequently, at the $\mathrm{GHz}$ range, it is more probable that polarization processes arise from electric dipolar polari zation rather than from electron or ion polarization. As fre quency increases, polarization of dielectrics becomes less influential because the formation of dipoles cannot follow the applied electric field and losses its responsiveness to it. As permittivity is a direct measure of the relationship be tween the polarization and the applied field [52], if a given polarization process loses its response, permittivity tends to decrease. In our case, for any given concentration, this effect is reflected in the decreasing trend observed for both the real and imaginary (not shown) parts of the permittivity with increasing frequency (Fig. 5).

At CNF concentrations above the percolation threshold, the electron tunnelling between adjacent conductive fibres is easier whenever the polymer insulating barrier between them is thinner. Increasing the mass fraction of CNF results in an increment in the real part of the permittivity, $\varepsilon^{\prime}$, because there are more polarisable charge transporters, meanwhile the conductivity increases due to favoured spatial displace ment of these currents. This observation is consistent with the behaviour observed for other carbon nanotube based composites [53,54].

Purely carbonaceous materials show microwave energy absorption mainly because of their characteristic dielectric loss properties. However, this may lead to an unbalanced 

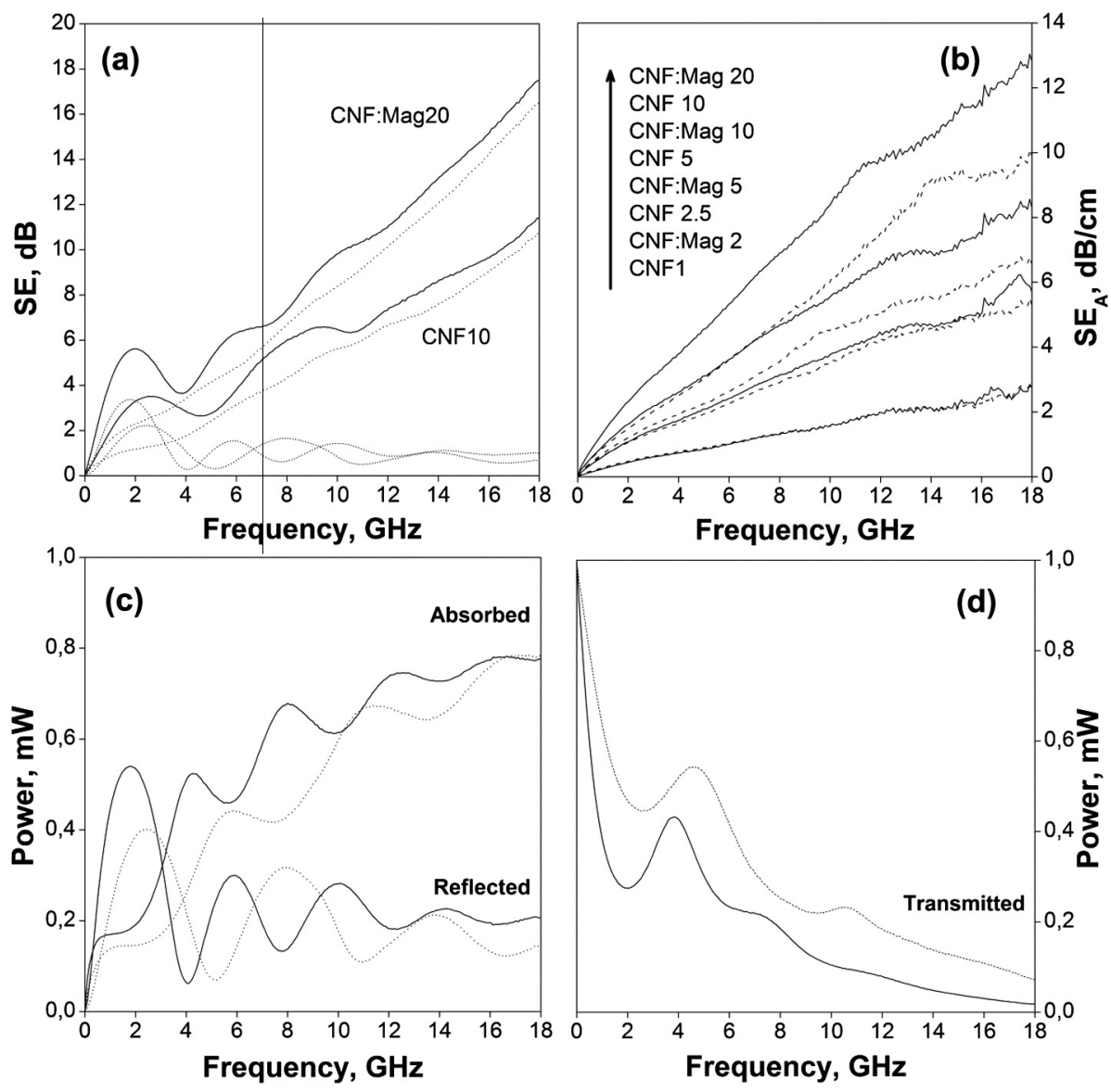

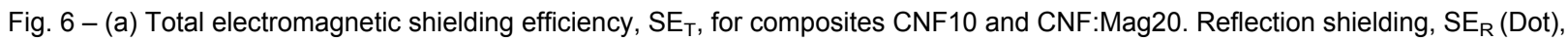
absorption shielding, $\mathrm{SE}_{\mathrm{A}}$ (Dash), transmission shielding, $\mathrm{SE}_{\mathrm{T}}$ (line). Thickness of specimens: $13 \mathrm{~mm}$. (b) Absorption coefficients for CNF (dashes), CNF:Mag (lines) composites with compositions indicated in the inset. (c) Absorbed, reflected and (d) transmitted power of CNF10 (dash) and CNF:Mag20 (line).

impedance matching condition, because of their lack of mag netic absorption [55]. This problem has been recently pro posed to be avoided by the incorporation of magnetic constituents within the carbonaceous structures [56], although the complex permeability values $\left(\mu^{\prime} \approx 1\right.$ and $\mu^{\prime \prime} \approx 0$ ) (see supplementary information) have led to contradictory conclusions about the magnetic loss irrelevancy when com pared to the dielectric loss. In this work, the above mentioned permeability values are close to one and zero as well as men tioned above, with some slight fluctuations. From this obser vation it may be possible to speculate that the incorporation of magnetite nanoparticles may not turn the fibres magneti cally active enough, but may be the responsible of an en hanced electric dipolar polarization process of the conducting fibres [57]. Following this hypothesis, when increasing the CNF:Mag loading there will be more charges prompt to polarization at the magnetite/CNF interfaces and the global polarization process would be accordingly stronger, thus favouring the charge transport along the nanofibres. It therefore appears that magnetite nanoparticles, when in elec tric contact with CNF, show a synergistic effect on both per mittivity and conductivity provided that the mass fraction of filler is high enough.

\subsection{Electromagnetic shielding}

Looking at the dielectric loss tangent values in Fig. 5, it can be seen that loses increase with concentration for both CNF and CNF:Mag systems. As the loss tangent is a direct evaluation of the attenuating properties of a given material, SE is expected to follow the same increasing trend with concentration; con sequently, because of their higher dielectric loss values, SE for CNF:Mag composites should be enhanced.

Contributions to SE were determined from the scattering parameters, using Eqs. (3) (5). Total shielding efficiency $\left(\mathrm{SE}_{\mathrm{T}}\right)$ as well as absorption $\left(\mathrm{SE}_{\mathrm{A}}\right)$ and reflection $\left(\mathrm{SE}_{\mathrm{R}}\right)$ components for the higher CNF compositions in both systems, CNF10 and CNF:Mag20, are shown in Fig. 6.

The most important feature in this graph is that above 3 $4 \mathrm{GHz}$, the main contribution to $\mathrm{SE}$ is absorption $\left(\mathrm{SE}_{\mathrm{A}}\right)$, while reflection $\left(\mathrm{SE}_{\mathrm{R}}\right)$ remains very low. Absorption contribution in creases with $f$, reaching a maximum value of $18 \mathrm{~dB}$ for CNF:Mag 20 and $12 \mathrm{~dB}$ for the one that only contains CNF. Concerning the reflection component, typical multiple max ima related to reflections from the second plane of the sam ple, which depend on sample thickness and, on a lesser extent, to the composition, are also observed. Nevertheless, 
above $4 \mathrm{GHz}$, reflection component remains at a very low level $(\sim 1 \mathrm{~dB})$.

The high attenuation presented by CNF:Mag, compared with CNF alone, is a clear demonstration of the synergistic ef fect of magnetite particles in electric contact with CNF. This effect is more clearly evidenced in Fig. 6, where the absorp tion coefficient, non dependent on sample thickness, is plot ted for all the prepared materials.

The absorption coefficient increases as the proportion of CNF in the composite increases and decoration of CNF with magnetite nanoparticles has a remarkable influence, espe cially at high contents. Recalling the discussion on DC con ductivity measurements, the presence of magnetite decreases the total contact area for electron hopping; but, as it became apparent in the discussion of dielectric results, it introduces an additional dissipation mechanism associated to the increased number of boundaries at the CNF/magnetite interface leading to an enhanced interfacial polarization, a fact that seems to be responsible for the observed synergy in the absorption shielding efficiency.

When increasing the concentration of conductive inclu sions in polymer composites, although several authors have reported absorption being the main internal shielding mech anism, as is the case herein presented, they do not commonly take into account that this discussion is assessed to the EM power that has not been reflected at the input interface of the slab $[58,59]$. Thus, evaluating the power balance of the in put EM wave is of primary importance when trying to deter mine whether the composite may be considered as a real EMI absorber, since reflection occurs before absorption. In or der to verify that composites prepared in this work might be considered as EMI absorbers, reflected $\left(P_{R} \quad\left|S_{11}\right|^{2}\right)$, transmitted $\left(\begin{array}{lllll}P_{\mathrm{T}} & \left|\mathrm{S}_{21}\right|^{2}\end{array}\right)$, absorbed $\left(\begin{array}{llll}P_{\mathrm{A}} & P_{\mathrm{I}} & P_{\mathrm{R}} & P_{\mathrm{T}}\end{array}\right)$ and incident $\left(P_{\mathrm{I}} \quad 1 \mathrm{~mW}\right)$ powers of samples showing the highest conductivities (CNF10 and CNF:Mag20), thus having the highest CNF/ CNF:Mag content, were plotted against frequency (Fig. 6).

Because power values are absolute values any factor affecting reflected power will directly influence the magni tude of the absorbed power. As shown in Fig. 6c, small differ ences in absorbed power are observed for the two samples. These are due to differences in reflected power. For example, at $17 \mathrm{GHz}$ reflected power in the sample CNF is lower than in the sample CNF:Mag20 but the absorbed power is the same for both samples. Although this result may appear to contra dict Fig. 6b, in which sample CNF:Mag20 clearly shows higher absorption, it simply indicates that less energy is entering the sample and, therefore, less energy is being absorbed. The ef fect of magnetite on the nanofibres can be clearly seen in Fig. 6d, where transmitted power is considerably lower for the sample containing magnetite.

\section{Conclusions}

In this work, we have prepared epoxy based composite mate rials loaded with variable amounts of CNF, magnetite nano particles and magnetite decorated CNF via solvent mixing procedures. Decoration of CNF has been achieved adsorbing preformed oleic acid capped magnetite nanoparticles onto their surface in a very simple and rapid way taking advantage of the hydrophobic nature of the CNF surface.
Powder X ray Diffraction has proved the preservation of the original crystallographic structure from both the magne tite nanoparticles and the pristine CNF during the decoration process. Good dispersion of these reinforcements in epoxy thermosets were obtained as indicated by their high conduc tivity values. The fact that both fillers (pristine and decorated CNF) present a similar percolation threshold indicates that adsorbed magnetite does not appreciably alter the dispersion process of the filler. DC conductivity measurements suggest that fluctuation tunnelling may be the underlying mecha nism for electron conduction, the gap width of decorated and non decorated CNF composites is similar and the ad sorbed magnetite particles reduce the contact area between the CNF.

The frequency dependence of permittivity and conductiv ity was explained in terms of the percolation theory in fractal structures. On the other hand, the high permittivity, the low magnetic permeability and the high loss tangent values indi cate that the behaviour of the composites is mainly related with the interaction with the electric field. Decoration of CNF with magnetite nanoparticles introduces interfacial polarization as an additional dissipation mechanism that is probably related with the synergistic behaviour in conductiv ity and permittivity observed at high loadings (CNF:Mag 20).

Contributions to the electromagnetic shielding efficiency and the power balance were determined from the scattering parameters, being the absorption contribution the most important in materials containing CNF and CNF:Mag, with low losses by reflection. The combination of CNF and magne tite nanoparticles in electrical contact seems to have also a synergistic effect on the shielding efficiency, yielding higher values than the ones obtained for composites containing only CNF and similar or better than those reported in literature as discussed in the introduction.

Decoration of nano conductive fillers with magnetic nano particles may be a suitable route to obtain materials with im proved shielding properties. This method is industrially scalable because of its simplicity, the amount of magnetite can be easily modified as required and it is not necessary to grow CNF using magnetic catalysts.

\section{Acknowledgments}

This work was supported by grants Nacopan (MAT2007 31173 E) and Nanomod (MAT2010 17091) from the Spanish Ministerio de Ciencia e Innovación. 


\section{R E F E R E N C E S}

[1] Schlechter M. EMI/RFI: Materials and Technologies [cited 2011 April 20]. Available from: <www.bccresearch.com>.

[2] Jiang G, Gilbert M, Hitt DJ, Wilcox GD, Balasubramanian K. Preparation of nickel coated mica as a conductive filler. Compos Part A Appl Sci Manuf 2002;33(5):745 51.

[3] Murthy M. Permanent EMI shielding of plastics using copper fibers annual technical conference ANTEC. San Francisco, CA, USA: Society of Plastics Engineers; 1994

[4] Al Saleh MH, Sundararaj U. A review of vapor grown carbon nanofiber/polymer conductive composites. Carbon 2009;47(1):2 22

[5] Antunes M, Mudarra M, Velasco JI. Broad band electrical conductivity of carbo' $n$ nanofibre reinforced polypropylene foams. Carbon 2011;49(2):708 17

[6] Yang S, Lozano K, Lomeli A, Foltz HD, Jones R. Electromagnetic interference shielding effectiveness of carbon nanofiber/ LCP composites. Compos Part A Appl Sci Manuf 2005;36(5):691 7.

[7] Ima JS, Kima JG, Leeb SH, Lee YS. Effective electromagnetic interference shielding by electrospun carbon fibers involving $\mathrm{Fe}_{2} \mathrm{O}_{3} / \mathrm{BaTiO}_{3} /$ MWCNT additives. Mater Chem Phys 2010;124(2 3):434 8

[8] Chen IH, Wang CC, Chen CY. Fabrication and characterization of magnetic cobalt ferrite/polyacrylonitrile and cobalt ferrite/carbon nanofibers by electrospinning. Carbon 2010;48(3):604 11

[9] Liu Z, Bai G, Huang Y, Ma Y, Du F, Li F, et al. Reflection and absorption contributions to the electromagnetic interference shielding of single walled carbon nanotube/polyurethane composites. Carbon 2007;45(4):821 7.

[10] Mahmoodi M, Arjmand M, Sundararaj U, Park S. The electrical conductivity and electromagnetic interference shielding of injection moulded multi walled carbon nanotube/polystyrene composites. Carbon 2012;50(4):1455 64.

[11] Yang Y, Gupta MC, Dudley KL, Lawrence RW. Novel carbon nanotube polystyrene foam composites for electromagnetic interference shielding. Nano Lett 2005;5(11):2131 4.

[12] Arjmand M, Mahmoodi M, Gelves GA, Park S, Sundararaj U.

Electrical and electromagnetic interference shielding properties of flow induced oriented carbon nanotubes in polycarbonate. Carbon 2011;49(11):3430 40 .

[13] Yun J, Im JS, Lee YS, Kim HI. Effect of oxyfluorination on electromagnetic interference shielding behavior of MWCNT/PVA/PAAC composite microcapsules. Eur Polym J 2010;46(5):900 9.

[14] Guadagno L, De Vivo B, Di Bartolomeo A, Lamberti P, Sorrentino A, Tucci V, et al. Effect of functionalization on the thermo mechanical and electrical behavior of multi wall carbon nanotube/epoxy composites. Carbon 2011;49(6):1919 30

[15] Qing Y, Zhou W, Luo F, Zhu D. Epoxy silicone filled with multi walled carbon nanotubes and carbonyl iron particles as a microwave absorber. Carbon 2010;48(14):4074 80.[16] Kim YJ,

Shin TS, Choi HD, Kwon JH, Chung Y, Yoon HG.

Electrical conductivity of chemically modified multiwalled carbon nanotube/epoxy composites. Carbon 2005;43(1):23 30.

[17] Feng QP, Yang JP, Fu SY, Mai YW. Synthesis of carbon nanotube/epoxy composite films with a high nanotube loading by a mixed curing agent assisted layer by layer method and their electrical conductivity. Carbon 2010;48(7):2057 62.

[18] Li N, Huang Y, Du F, He X, Lin X, Gao H, et al. Electromagnetic interference (EMI) shielding of single walled carbon nanotube epoxy composites. Nano Lett 2006;6(6):1141 5.[19] Che RC,

Zhia CY, Liang CY, Zhou XG. Fabrication and microwave absorption of carbon nanotubes/ $/ \mathrm{CoFe}_{2} \mathrm{O}_{4}$ spinel composite. Appl Phys Lett 2006;88(3):033105.
[20] Singh AP, Garg P, Alam F, Singh K, Mathur RB, Tandon RP, et al. Phenolic resin based composite sheets filled with mixtures of reduced graphene oxide, $\gamma \mathrm{Fe}_{2} \mathrm{O}_{3}$ and carbon fibers for excellent electromagnetic interference shielding in the X band. Carbon 2012;50(10):3868 75.

[21] Yang Y, Gupta MC, Dudley KL. Towards cost efficient EMI shielding materials using carbon nanostructure based composites. Nanotechnology 2007;18:345701.

[22] Chung DDL. Electromagnetic interference shielding effectiveness of carbon materials. Carbon 2001;39(2):279 85

[23] Al Saleh MH, Sundararaj U. Electromagnetic interference shielding mechanisms of CNT/polymer composites. Carbon 2009;47(7):1738 46.

[24] May CA. Epoxy resins, chemistry and technology. New York: Marcel Dekker; 1988.

[25] Klaassens LI, De Jong J, Gillard M, Van der Poel H. European Patent EP 1359197A1, 2003.

[26] Klaassens LI, De Jong J, Van der Poel H, European Patent EP 1359198A1; 2003.

[27] Mowrer NR, Foscante RE, Rojas L. United States Patent US 5618860; 1997.

[28] Mowrer NR, Foscante RE, Rojas L. United States Patent US 5804616; 1998

[29] Gonza' lez M, Martı́n Fabiani I, Baselga J, Pozuelo J. Magnetic composites based on hydrogenated epoxy resin. Mater Chem Phys 2012;132(2 3):618 24

[30] Cipiriano BH, Kota AK, Gershon AL, Laskowski CJ, Kashiwagi $\mathrm{T}$, Bruck HA, et al. Conductivity enhancement of carbon nanotube and nanofiber based polymer composites by melt annealing. Polymer 2008;49(22):4846 51.

[31] Smrutisikha B. Experimental study of mechanical and electrical properties of carbon nanofiber/epoxy composites. Mater Des 2010;31(5):2406 13.

[32] Stauffer D. Introduction to percolation theory. London: Taylor \& Francis; 1987. p. 89.

[33] Connor MT, Roy S, Ezquerra TA, Balta' Calleja FJ. Broadband ac conductivity of conductor polymer composites. Phys Rev B Condens Matter 1998;57(4):2286 94.

[34] Sheng P, Sichel EK, Gittleman JI. Fluctuation induced tunneling conduction in carbon polyvinylchloride composites. Phys Rev Lett 1978;40(18):1197 200.

[35] Sheng P. Fluctuation induced tunnelling conduction in disordered materials. Phys Rev B Condens Matter 1980;21(6):2180 95.

[36] Marand E, Baker KR, Graybeal JD. Comparison of reaction mechanisms of epoxy resins undergoing thermal and microwave cure from in situ measurements of microwave dielectric properties and infrared spectroscopy. Macromolecules 1992;25(8):2243 52.

[37] Mijovic J, Fishbain A, Wijaya J. Mechanistic modelling of epoxy amine kinetics. 2. Comparison of kinetics in thermal and microwave fields. Macromolecules 1992;25(2):986 9.

[38] Mijovic J, Han Y, Sun M, Pejanovic S. Networks undergoing

chemical cross linking. Epoxy/amine terminated linear and star PPO formulations. Macromolecules 2003;36(12):4589 602 .

[39] Fitz BD, Mijovic J. Segmental dynamics and density fluctuations in polymer networks during chemical vitrification. Macromolecules 1999;32(12):4134 40.

[40] Ali zade RA. Permittivity of nanocomposites based on magnetite nanoparticles and polymer matrices (collagen and polystyrene). Russ J Phys Chem A 2010;84(9):1570 5

[41] Balberg I. Recent developments in continuum percolation. Philos Mag A 1987;56(6):991 1003.

[42] Balberg I, Anderson CH, Alexander S, Wagner N. Excluded volume and the onset of percolation. Phys Rev B Condens Matter 1984;30(7):3933 
[43] Miller MA. On structural correlations in the percolation of hard core particles. J Chem Phys 2009;131(6):2009.

[44] Prasad A, Prasad K. Effective permittivity of random composite media: a comparative study. Physica B 2007;396(1 2):132 7 .

[45] Fannin PC, Marin CN, Malaescu I, Stefu N. Microwave

dielectric properties of magnetite colloidal particles in magnetic fluids. J Phys Condens Matter 2007;19:036104.

[46] Li B, Sui G, Zhong WH. Single negative metamaterials in unstructured polymer nanocomposites toward selectable and controllable negative permittivity. Adv Mater 2009;21(41):4176 80.

[47] Sui G, Li B, Bratzel G, Baker L, Zhong W H, Yang X P. Carbon nanofiber/polyetherimide composite membranes with special dielectric properties. Soft Mater 2009;5(19):3593 8.[48] Terrones M,

Martín O, Gonza' lez M, Pozuelo J, Serrano B,

Cabanelas JC, et al. Interphases in graphene polymer based composites: achievements and challenges. Adv Mater 2011;23(44):5302 10.

[49] Laibowitz RB, Gefen Y. Dynamic scaling near the percolation threshold in thin Au films. Phys Rev Lett 1984;53(4):380 3.[50] Gefen Y,

Aharony A, Alexander S. Anomalous diffusion on percolating clusters. Phys Rev Lett 1983;50(1):77 80

[51] Bo"ttger U. Dielectric properties of polar oxides. In: Waser R, Bo"ttger U, Tiedke S, editors. Polar oxides: properties, characterization, and imaging. Weinheim: Wiley VCH Verlag $\mathrm{GmbH} \&$ Co. KGaA; 2005.

[52] Labunov VA, Danilyuk AL, Prudnikava AL, Komissarov I, Shulitski BG. Microwave absorption in nanocomposite material of magnetically functionalized carbon nanotubes. J Appl Phys 2012;112(2):024302.

[53] Liu Z, Bai G, Huang Y, Ma Y, Du F, Li F, et al. Reflection and

absorption contributions to the electromagnetic shielding of single walled carbon nanotube/polyurethane composites. Carbon 2007;45(4):821 7.

[54] Singh AP, Gupta BK, Mishra M. Govind, Chandra A, Mathur RB, Dhawan SK. Multiwalled carbon nanotube/cement composites with exceptional electromagnetic interference shielding properties. Carbon 2013;56:86 96.

[55] Ramirez AP, Haddon RC, Zhou O, Fleming RM, Zhang J, McClure SM, et al. Magnetic susceptibility of molecular carbon: nanotubes and fullerite. Science 1994;265(5168):84 6.[56] Zhang X,

Liu Y, Qin J. The dielectric properties of ( $\mathrm{N}$ doped carbon) and carbon coated iron nanocrystals in the $\mathrm{X}$ band. Carbon 2004;42(4):888 90

[57] Jiang M, Dang Z, Bozlar M, Miomandre F, Bai J. Broad frequency dielectric behaviors in multiwalled carbon nanotube/ rubber nanocomposites. J Appl Phys 2009;106(8):084902 8.

[58] Huynen I, Quie'vy N, Bailly C, Bollen P, Detrembleur C, Eggermont S, et al. Multifunctional hybrids for electromagnetic absorption. Acta Mater 2011;59(8):3255 66.[59] Quie'vy N, Bollen P, Thomassin JM, Detrembleur C, Pardoen T,

Bailly C, et al. Electromagnetic absorption properties of carbon nanotube nanocomposite foam filling honeycomb waveguide structures. IEEE Trans Electromagn Compat 2012;54(1):43 51 